\title{
Nonspecific pain is a marker for hypovitaminosis $D$ in patients undergoing evaluation for sleep disorders: a pilot study
}

This article was published in the following Dove Press journal:

Nature and Science of Sleep

8 March 2013

Number of times this article has been viewed

\section{David E McCarty' \\ Aronkumar Reddy' \\ Quinton Keigley ${ }^{2}$ \\ Paul Y Kim' \\ Stephen Cohen² \\ Andrew A Marino' \\ 'Division of Sleep Medicine,} Department of Neurology, Louisiana State University Health Sciences Center, ${ }^{2}$ School of Medicine, Louisiana State University, Shreveport, LA, USA

Correspondence: David E McCarty Department of Neurology,

LSUHSC-Shreveport, PO Box 33932,

I50I Kings Highway, Shreveport,

LA 7II 30-3932, USA

Tel + I 3186758568

Fax +I 3186754440

Email dmcca I@Isuhsc.edu
Background: The purpose of this cross-sectional study was to test the hypothesis that serum vitamin D levels are abnormally low in sleep clinic patients admitting to chronic nonspecific musculoskeletal pain and to assess the associated risk factors. A secondary purpose was to identify a clinical biomarker for vitamin D deficiency.

Methods: We enrolled 153 consecutive patients who admitted to the presence of chronic nonspecific musculoskeletal pain during a comprehensive sleep evaluation at a specialist sleep medicine clinic within an academic center. Venous blood sampling was performed for determination of serum 25-hydroxyvitamin D. Risk factors for vitamin D deficiency (25-hydroxyvitamin $\mathrm{D}<20 \mathrm{ng} / \mathrm{mL}$ ) were identified by odds ratios. Receiver-operating characteristic curve analysis was performed with 10 -fold cross-validation to identify a biomarker for vitamin D deficiency calculated by linear discriminant analysis.

Results: The mean serum 25-hydroxyvitamin D level was $19.8 \pm 11.1$, with $54 \%$ of the study population having vitamin D deficiency. This mean 25-hydroxyvitamin D level was lower than that observed historically in healthy controls, and was either similar or lower than in all but one representative historical cohort formed on the basis of chronic nonspecific musculoskeletal pain. Risk factors for vitamin D deficiency were black ethnicity, age $<60$ years, and obesity. These risk factors were identified both in the entire cohort and separately in subgroups with and without obstructive sleep apnea. The biomarker (based on race, age, and body mass index) had a sensitivity and specificity for predicting vitamin D deficiency of 0.73 and 0.74 , respectively.

Conclusion: Vitamin D deficiency was prevalent in patients with sleep disorders and chronic nonspecific musculoskeletal pain on evaluation in a sleep medicine clinic. Vitamin D deficiency was reliably estimated in the study population using a biomarker derived from common demographic characteristics.

Keywords: vitamin D, biomarker, pain, sleep, linear discriminant analysis, receiver-operating analysis

\section{Introduction}

Vitamin D is a fat-soluble secosteroid prohormone ingested in the diet and produced in the skin following exposure to ultraviolet rays in sunlight, and conversion to active forms of vitamin D occurs in the liver and kidneys. ${ }^{1}$ The metabolic processes regulated by vitamin D include serum calcium and phosphate homeostasis, bone remodeling, neuromuscular function, inflammation, and transcription of proteins involved in cell growth and apoptosis. ${ }^{2}$ Nonspecific musculoskeletal pain and weakness are prominent features of many diseases linked to vitamin D deficiency. ${ }^{3-6}$ Vitamin D deficiency may increase the risk of developing obstructive sleep apnea mediated 
by inflammatory rhinitis ${ }^{7,8}$ and/or tonsillar hypertrophy. ${ }^{9,10}$ Vitamin D deficiency may also contribute to the development of daytime sleepiness via central inflammatory mediators. $^{11,12}$

Patients seeking care in a sleep medicine clinic typically complain of sleep disruption, fatigue, and sleepiness, sometimes in association with chronic nonspecific musculoskeletal pain. Low levels of serum vitamin $\mathrm{D}$ have been reported in patients who report such pain, ${ }^{4,13}$ but it is not clear if these data apply to patients whose pain is discovered incidentally during evaluation for another complaint. We were interested in the possibility that the presence of pain might indicate low vitamin D levels contributing to poor sleep quality and/ or daytime symptoms of impairment in patients undergoing evaluation for a suspected sleep disorder.

Traditional risk factors for low vitamin D include race (with darker skin tone conferring higher risk), obesity, and advancing age. ${ }^{1}$ Our primary aim was to test the hypothesis that subnormal levels of vitamin D exist in patients with sleep disorders who admit to chronic nonspecific musculoskeletal pain, and to evaluate whether predictive risk factors were present. Our secondary aim was to formulate a biomarker for vitamin $\mathrm{D}$ deficiency based on the risk factors observed.

\section{Materials and methods Patients}

Between April, 2008 and October, 2010, consecutive patients seen during routine consultation visits at an academic sleep medicine clinic were interviewed within the context of a full sleep history and underwent a physical examination. The patients also completed a questionnaire screening for the presence of various symptoms potentially linked to sleep disruption, including the presence of moderate to severe musculoskeletal pain contributing to sleep disruption or daytime discomfort, or both. All questionnaire responses were subsequently reviewed and verified in a face-to-face interview by a physician board-certified in internal medicine and sleep medicine (DM). For some patients, pain symptoms were initially denied, but were disclosed after specific questioning about regions of the body where pain might be experienced (eg, "Do you have pain in your back, legs, or arms that you notice during sleep?"). Patients who admitted to the presence of functionally significant pain and agreed to undergo venous blood sampling for 25-hydroxyvitamin D $(n=153)$ were included in the analysis (Table 1). All research-related procedures were approved by the institutional review board for human research at our institution.
Table I Characteristics of cohort $(n=153)$

\begin{tabular}{ll}
\hline Age (years) & $50.9 \pm 13.7$ \\
Gender (\%) & \\
Male & 23.5 \\
Female & 76.5 \\
Race $(\%)$ & \\
African-American & 35.3 \\
Caucasian/Hispanic* & 64.7 \\
Body mass index $\left(\mathrm{kg} / \mathrm{m}^{2}\right)$ & $36.1 \pm 9.8$ \\
$\geq 30$ & 72 \\
$<30$ & 28 \\
Sleep diagnosis $(\%)$ & \\
Obstructive sleep apnea & 77 \\
Hypersomnia & 8.5 \\
Insomnia & 15 \\
Restless legs syndrome & 28 \\
Other & 6.5 \\
\hline
\end{tabular}

Notes: Values are presented as the mean \pm standard deviation; *Hispanic subjects $\mathrm{n}=2$.

\section{Serum measurements}

Serum 25-hydroxyvitamin D levels were determined by immunoassay according to the specifications of the manufacturer (DiaSorin Liason, Saluggia, Italy). A 25-hydroxyvitamin D level below $20 \mathrm{ng} / \mathrm{mL}$ was considered to indicate vitamin D deficiency. $^{1}$

\section{Study design and statistical analysis}

The study design is shown in Figure 1. The unpaired $t$-test was used to compare mean 25-hydroxyvitamin D levels, with reported representative mean values for normal subjects and for groups formed on the basis of a primary complaint of somatic pain. The relationship between vitamin $\mathrm{D}$ deficiency and known risk factors was evaluated by computing odds ratios and 95\% confidence limits. The risk factors considered were race (black versus white/Hispanic), obesity (body mass index $\leq 30 \mathrm{~kg} / \mathrm{m}^{2}$ versus $>30 \mathrm{~kg} / \mathrm{m}^{2}$ ), age ( $\leq 60$ years versus $>60$ years), gender, and season (blood draw months during summer [May-June-July] versus winter [NovemberDecember-January]). Mean 25-hydroxyvitamin D values were analyzed for the entire cohort and according to significant risk factors.

A biomarker function for predicting vitamin D deficiency was derived using linear discriminant analysis. ${ }^{14}$ Its predictive accuracy was assessed by calculating sensitivity, specificity, and area under the receiver-operator characteristics curve (AUROC). ${ }^{15}$ The likelihood that the results would be able to be generalized was estimated by computing a biomarker function using data from $90 \%$ of the patients and evaluating it using data from the remaining $10 \%$ of patients. The procedure was repeated ten times using different groups 


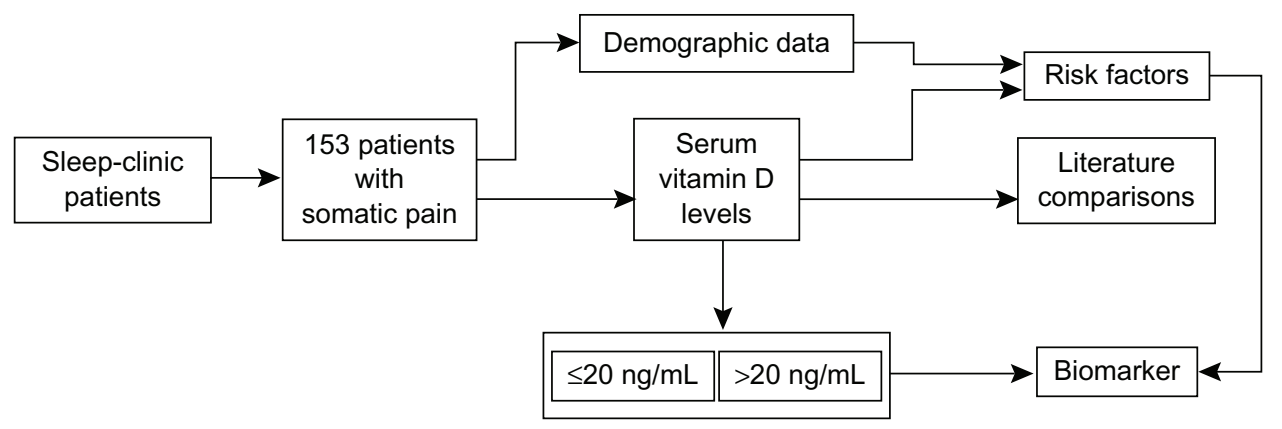

Figure I Study flow chart.

of ten patients to evaluate the predictive accuracy. Overall accuracy was estimated by averaging the results of the ten validation procedures.

\section{Results}

The mean 25-hydroxyvitamin D level in the study population was $19.8 \pm 11.1 \mathrm{ng} / \mathrm{mL}$, with $54 \%$ of the patients having vitamin $\mathrm{D}$ deficiency (25-hydroxyvitamin D level $<20 \mathrm{ng} / \mathrm{mL}$ ). This 25 -hydroxyvitamin D level was significantly lower than that commonly seen in clinically normal populations, and the prevalence of vitamin D deficiency was consistently higher (Table 2 ). Compared with the results of published studies involving patients for whom pain was a primary complaint, the mean level in our cohort was

Table 2 Comparison of serum levels of 25 -hydroxyvitamin D in our study group with those published for normal subjects and those with somatic pain

\begin{tabular}{lcll}
\hline $\mathbf{n}$ & $\begin{array}{l}\text { 25-hydroxy } \\
\text { vitamin D }(\mathbf{n g} / \mathbf{m L})\end{array}$ & $\begin{array}{l}\text { Percent } \\
\text { deficient }\end{array}$ & Reference \\
\hline 153 & $19.8 \pm I I . I$ & $54 \%$ & This study \\
Clinically & normal subjects & & \\
493 & $* 34.2 \pm 13.8$ & NR & 19 \\
212 & $* 23.3 \pm 8.4$ & $40 \%$ & 20 (Winter) \\
99 & $* 26.8 \pm 10.3$ & $20 \%$ & 20 (Summer) \\
165 & $* 30 \pm 10$ & $30 \%$ & 21 (Winter) \\
142 & $* 35 \pm 10$ & $12 \%$ & 21 (Summer) \\
202 & $* 33.1 \pm 28.4$ & $36 \%$ & 22 \\
13,369 & $* 24 \pm 1$ & $36 \%$ & 23 \\
Patients with pain & & \\
128 & $* 28.6 \pm 13.2$ & NR & 19 \\
150 & $* 12.1 \pm 5.8$ & $93 \%$ & 24 \\
267 & $* 28.7 \pm 11.6$ & $26 \%$ & 25 \\
276 & $23.8 \pm 29.1$ & $63 \%$ & 22 \\
25 & $20.6 \pm 6.8$ & $N R$ & 26 \\
572 & $18.8 \pm 1.0$ & $58 \%$ & 27 \\
263 & $* 23.9 \pm 11.8$ & $26 \% \ddagger$ & 28 \\
\hline
\end{tabular}

Notes: Data are presented as the mean \pm standard deviation. Vitamin $D$ deficiency was defined as a serum concentration of 25 -hydroxyvitamin $D<20 \mathrm{ng} / \mathrm{mL}$ except for ${ }^{\ddagger}$ which indicates $\leq 15 \mathrm{ng} / \mathrm{mL}$. $* P<0.05$, $t$-test.

Abbreviation: NR, not reported. either significantly lower or statistically indistinguishable, with only one exception (Table 2).

The risk for vitamin $\mathrm{D}$ deficiency was 50 times higher in black than in white/Hispanic subjects, and an increased risk was also seen in subjects who were obese or younger than 60 years (Table 3). Increased risk was not observed in association with gender or winter months. In subjects from whom blood was collected during the summer months, 23 of $41(56 \%)$ had vitamin D deficiency compared with 18 of 31 subjects (58\%) from whom blood was collected during the winter months. Mean 25-hydroxyvitamin D levels in patients with and without obstructive sleep apnea were not significantly different (Table 4). Risk factors for vitamin D deficiency (Table 3) were generally found to be predictive in patients with and without obstructive sleep apnea (Table 4).

The biomarker function formed on the basis of the risk factors found to be associated with vitamin $\mathrm{D}$ deficiency was $\mathrm{B}(\mathrm{x})=0.207 \mathrm{x}_{1}+0.011 \mathrm{x}_{2}-0.007 \mathrm{x}_{3}+0.092$, where $\mathrm{x}_{1}$ is race ( $1=$ white/Hispanic, 2 = black), $\mathrm{x}_{2}$ is body mass index $\left(\mathrm{kg} / \mathrm{m}^{2}\right)$, and $\mathrm{x}_{3}$ is age (years). The AUROC was 0.78, and the mean AUROC for ten 90:10 cross-validation determinations was $0.75 \pm 0.02$. At a biomarker threshold value of 0.45 , the sensitivity and specificity were 0.73 and 0.74 , respectively. The performance of the biomarker function in classifying each patient in the cohort is shown in Figure 2.

Table 3 Association between known risk factors and vitamin $D$ deficiency (25-hydroxyvitamin $\mathrm{D}<20 \mathrm{ng} / \mathrm{mL}$ ) in the study group

\begin{tabular}{ll}
\hline Factor & Odds ratio (95\% CI) \\
\hline Race (African-American/Caucasian or Hispanic) & $50.8^{*}(\mathrm{I} 5.3-\mathrm{I} 68.4)$ \\
Obesity (BMI $\left.>30 \mathrm{~kg} / \mathrm{m}^{2}\right)$ & $2.87^{*}(\mathrm{I} .4-5.8)$ \\
Age (<60 years) & $2.5^{*}(\mathrm{I} .2-5 . \mathrm{I})$ \\
Gender & $1.2(0.6-2.4)$ \\
Season (winter/summer) & $1.2(0.6-7.5)$ \\
\hline
\end{tabular}

Note: $* P<0.05$, Chi-square test.

Abbreviations: $\mathrm{BMI}$, body mass index; $\mathrm{Cl}$, confidence interval. 
Table 4 Vitamin D levels and diagnosis stratified by ethnicity, obesity, and age

\begin{tabular}{|c|c|c|}
\hline & Black & White \\
\hline OSA & $* 13.2 \pm 7.4(43)$ & $22.7 \pm 10.9(75)$ \\
\hline \multirow[t]{2}{*}{ Non-OSA } & $* 15.0 \pm 9.1(11)$ & $25.3 \pm 12.4(24)$ \\
\hline & BMI $>30 \mathrm{~kg} / \mathrm{m}^{2}$ & $\mathrm{BMI} \leq \mathbf{3 0} \mathrm{kg} / \mathrm{m}^{2}$ \\
\hline OSA & $18.3 \pm 10.3(88)$ & $22.1 \pm 11.6(30)$ \\
\hline \multirow[t]{2}{*}{ Non-OSA } & $* 15.8 \pm 10.5(16)$ & $27.3 \pm 11.5(19)$ \\
\hline & Age $<60$ years & Age $\geq 60$ years \\
\hline OSA & $* 16.4 \pm 8.3(80)$ & $25.2 \pm 12.7(38)$ \\
\hline \multirow[t]{2}{*}{ Non-OSA } & $* 20.0 \pm 1 \mathrm{I} .4(3 \mathrm{I})$ & $38.1 \pm 6.6(4)$ \\
\hline & Entire cohort & \\
\hline OSA & $19.2 \pm 10.7(118)$ & \\
\hline Non-OSA & $22.1 \pm 12.3(35)$ & \\
\hline
\end{tabular}

Notes: Number of patients shown in parentheses; data are presented as the mean \pm standard deviation; vitamin $D$ levels are presented as $\mathrm{ng} / \mathrm{mL}$. $* P<0.05$. Abbreviations: BMI, body mass index; OSA, obstructive sleep apnea.

25-Hydroxyvitamin D levels were not significantly different between patients with and without obstructive sleep apnea. The biomarker function was found to be applicable for both groups (Table 4).

\section{Discussion}

Vitamin D is an ubiquitous metabolic regulator, and sleep is a physiologic process that can be impaired as a consequence of dysregulation of many different metabolic processes. We found that the presence of nonspecific functionally significant pain (revealed on direct questioning, but not necessarily

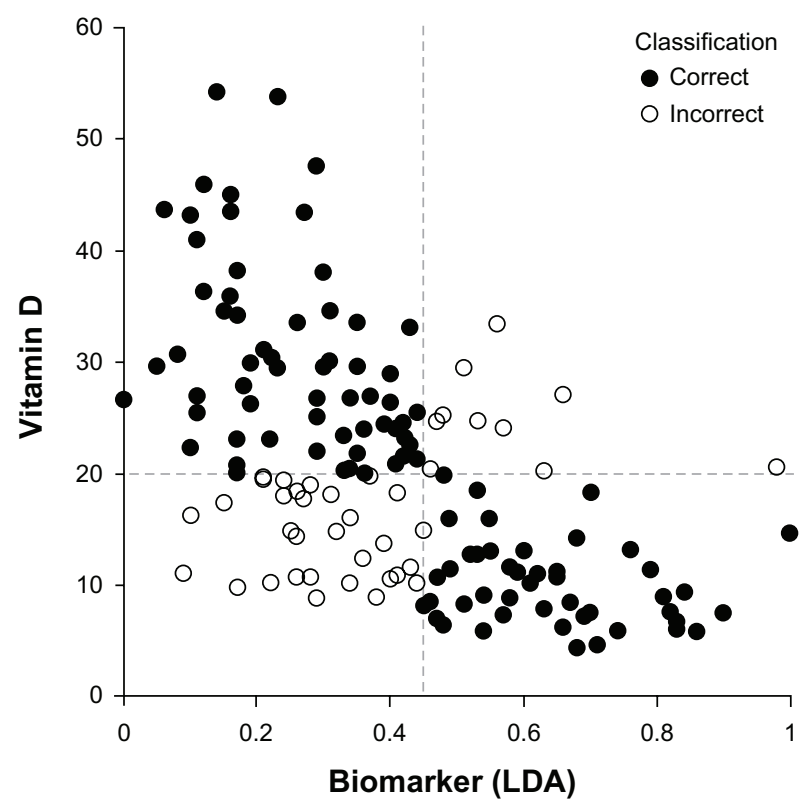

Figure 2 Correlation between vitamin $\mathrm{D}$ and biomarker by LDA based on age, race, and body mass index. Pearson correlation coefficient $r=0.505$.

Abbreviation: LSA, linear discriminant analysis. mentioned a priori by the patient) is a reliable marker for the presence of low vitamin $\mathrm{D}$ in patients undergoing specialist evaluation for a suspected sleep disorder.

Although low 25-hydroxyvitamin D has been previously reported in patients with complaints of chronic pain, patients in the cohort described in our study were incidentally discovered to have pain symptoms during consultation for another reason. In some cases, pain symptoms were disclosed only after specific questioning. Nevertheless, the 25-hydroxyvitamin $\mathrm{D}$ values found mirror the low values reported in cohorts defined on the basis of pain as a chief complaint, suggesting that 25-hydroxyvitamin D may play a role in the pathophysiology of diseases typically classified as sleep disorders. One possibility is that chronic nonspecific musculoskeletal pain contributes to subjective sleep disturbances and/or a magnification of perceived daytime impairment, and both symptoms frequently lead to specialist evaluation for a suspected sleep disorder.

Vitamin D deficiency has the biologic potential to contribute mechanistically to obstructive sleep apnea via myopathy, ${ }^{16}$ facilitating development of chronic rhinitis, ${ }^{7,8}$ or contributing to tonsillar hypertrophy. ${ }^{10}$ In our cohort, mean 25-hydroxyvitamin $\mathrm{D}$ values were not significantly different between those with and without obstructive sleep apnea $(P=0.17)$, and the risk factors identified for vitamin $\mathrm{D}$ deficiency were generally valid for both groups (Table 4). We have previously described a patient presenting with a syndrome clinically indistinguishable from idiopathic central nervous system hypersomnia whose excessive daytime sleepiness symptoms resolved following treatment of severe vitamin D deficiency, ${ }^{17}$ and we have also recently reported a relationship between Epworth Sleepiness Scale scores and 25-hydroxyvitamin D levels. ${ }^{18}$ Taken together, the evidence supports the notion that the presence of nonspecific pain in a patient with a suspected sleep disorder may be predictive of vitamin D deficiency, a problem which could act as a cofactor for perceived symptoms of a broad array of sleep disorders.

Although the present evidence does not warrant routine testing of all sleep clinic patients for vitamin D deficiency, a biomarker that reliably predicts vitamin $\mathrm{D}$ deficiency might form a useful basis for selecting patients for testing, ultimately leading to improved diagnosis and treatment. In the present study, the vitamin D status of a considerable number of our patients (vitamin D deficiency present or absent) was correctly identified based solely on standard demographic data (Figure 2). The presence or absence of 
obstructive sleep apnea did not appreciably alter the predictive value of the biomarker (Table 4).

An unexpected finding in this cohort was that younger age appeared to confer a higher risk for vitamin D deficiency. Typically, older age is associated with decreased ability of the skin to produce active vitamin D. Our current data do not provide a satisfactory explanation for this paradox. Age-dependent sun exposure habits, use of supplements, and use of medications (such as anticonvulsants or steroids) are known to decrease circulating 25-hydroxyvitamin D, and may be responsible. It is also possible that the nonspecific physical symptoms associated with vitamin D deficiency prompt younger patients to seek specialist care for sleep disorders. Further research is needed to clarify the relative contribution of each of these factors.

It might be argued that a potential limitation of this study was the absence of a control group without chronic nonspecific musculoskeletal pain. Because our main purpose was to compare our cohort with a healthy adult population and with populations formed on the basis of musculoskeletal pain as a chief complaint, we consider that use of historical controls was reasonable and clinically relevant for comparison purposes. Notwithstanding the putative limitation of historical control groups, the consistency and size of the observed differences (Table 2) indicate that the vitamin D levels found in our study were unusually low. The reliability of this inference is further supported by our finding that two risk factors strongly associated with vitamin D deficiency, ie, race and obesity, were also associated with vitamin D deficiency in the cohort (Table 2).

\section{Conclusion}

Sleep clinic patients who incidentally disclosed chronic nonspecific musculoskeletal pain during specialist evaluation at our sleep medicine clinic had significantly lower serum levels of 25-hydroxyvitamin D and a higher prevalence of vitamin $\mathrm{D}$ deficiency than historical controls without pain, and had 25-hydroxyvitamin D levels and vitamin D deficiency rates similar to patient groups defined on the basis of chronic pain as a presenting complaint. The likelihood of vitamin D deficiency in this cohort was estimated reliably using a biomarker derived from common demographic data. Chronic nonspecific musculoskeletal pain may be a symptom of hypovitaminosis D.

\section{Disclosure}

The authors report no conflicts of interest in this work.

\section{References}

1. Holick MF. Vitamin D deficiency. N Engl J Med. 2007;357(3): 266-281.

2. Holick MF. High prevalence of vitamin D inadequacy and implications for health. Mayo Clin Proc. 2006;81(3):353-373.

3. Glerup H, Mikkelsen K, Poulsen L, et al. Hypovitaminosis D myopathy without biochemical signs of osteomalacic bone involvement. Calcif Tissue Int. 2000;66(6):419-424.

4. Heidari B, Shirvani JS, Firouzjahi A, Heidari P, Hajian-Tilaki KO. Association between nonspecific skeletal pain and vitamin D deficiency. Int J Rheum Dis. 2010;13(4):340-346.

5. Holick MF. Vitamin D deficiency: what a pain it is. Mayo Clin Proc. 2003;78(12):1457-1459.

6. Russell JA. Osteomalacic myopathy. Muscle Nerve. 1994;17(6): 578-580.

7. Wjst M, Hypponen E. Vitamin D serum levels and allergic rhinitis. Allergy. 2007;62(9):1085-1086.

8. Abuzeid W, Akbar N, Zacharek M. Vitamin D and chronic rhinitis. Curr Opin Allergy Clin Immunol. 2012;12(1):13-17.

9. Grant WB. Tonsillectomy may be an indicator of low vitamin D status, a risk factor for cancer later in life. Cancer Causes Control. 2009;20(7):1235-1236.

10. Reid D, Morton R, Salkeld L, Bartley J. Vitamin D and tonsil disease - preliminary observations. Int J Pediatr Otorhinolaryngol. 2011;75(2):261-264.

11. McCarty DE. Resolution of hypersomnia following identification and treatment of vitamin D deficiency. J Clin Sleep Med. 2010;6(6): 605-608.

12. Hoeck AD, Pall ML. Will vitamin D supplementation ameliorate diseases characterized by chronic inflammation and fatigue? Med Hypotheses. 2011;76(2):208-213.

13. Plotnikoff GA, Quigley JM. Prevalence of severe hypovitaminosis D in patients with persistent, nonspecific musculoskeletal pain. Mayo Clin Proc. 2003;78(12):1463-1470.

14. Krzanowkski WJ. Principles of Multivariate Analysis: A User's Perspective. Revised Edition. New York, NY: Oxford University Press; 2000.

15. Zhou X-H, Obuchowski NA, McClish DK. Statistical Methods in Diagnostic Medicine. New York, NY: Wiley \& Sons; 2002.

16. Prabhala A, Garg R, Dandona P. Severe myopathy associated with vitamin D deficiency in western New York. Arch Intern Med. 2000;160(8):1199-1203.

17. McCarty DE. Resolution of hypersomnia following identification and treatment of vitamin D deficiency. J Clin Sleep Med. 2010;6(6):605-608.

18. McCarty DE, Reddy A, Keigley Q, Kim PY, Marino AA. Vitamin D, race, and excessive daytime sleepiness. J Clin Sleep Med. 2012;8(6): 693-697.

19. Ahmed W, Khan N, Glueck CJ, et al. Low serum $25(\mathrm{OH})$ vitamin D levels $(<32 \mathrm{ng} / \mathrm{mL})$ are associated with reversible myositis-myalgia in statin-treated patients. Transl Res. 2009;153(1):11-16.

20. Levis S, Gomez A, Jimenez C, et al. Vitamin D deficiency and seasonal variation in an adult South Florida population. J Clin Endocrinol Metab. 2005;90(3):1557-1562.

21. Tangpricha V, Pearce EN, Chen TC, Holick MF. Vitamin D insufficiency among free-living healthy young adults. Am JMed.2002;112(8): 659-662.

22. Heidari B, Shirvani JS, Firouzjahi A, Heidari P, Hajian-Tilaki KO. Association between nonspecific skeletal pain and vitamin D deficiency. Int J Rheum Dis. 2010;13(4):340-346.

23. Ginde AA, Liu MC, Camargo CA Jr. Demographic differences and trends of vitamin D insufficiency in the US population, 1988-2004. Arch Intern Med. 2009;169(6):626-632.

24. Plotnikoff GA, Quigley JM. Prevalence of severe hypovitaminosis D in patients with presistent, nonspecific musculoskeletal pain. Mayo Clinic Proc. 2003;78(12):1463-1470. 
25. Turner MK, Hooten WM, Schmidt JE, Kerkvliet JL, Townsend CO, Bruce BK. Prevalence and clinical correlates of vitamin D inadequacy among patients with chronic pain. Pain Med. 2008;9(8): 979-984.

26. Huisman AM, White KP, Algra A, et al. Vitamin D levels in women with systemic lupus erythematosus and fibromyalgia. $J$ Rheumatol. 2001;28(11):2535-2539.
27. Knutsen KV, Brekke M, Gjelstad S, Lagerløv P. Vitamin D status in patients with musculoskeletal pain, fatigue and headache: a crosssectional descriptive study in a multi-ethnic general practice in Norway. Scand J Prim Health Care. 2010;28(3):166-171.

28. McBeth J, Pye SR, O’Neill TW, et al. Musculoskeletal pain is associated with very low levels of vitamin D in men: results from the European Male Ageing Study. Ann Rheum Dis. 2010;69(8):1448-1452.

\section{Publish your work in this journal}

Nature and Science of Sleep is an international, peer-reviewed, open access journal covering all aspects of sleep science and sleep medicine, including the neurophysiology and functions of sleep, the genetics of sleep, sleep and society, biological rhythms, dreaming, sleep disorders and therapy, and strategies to optimize healthy sleep. The journal welcomes

\section{Dovepress}

original research, clinical \& epidemiological studies, reviews \& evaluations, case reports and extended reports. The manuscript management system is completely online and includes a very quick and fair peerreview system, which is all easy to use. Visit http://www.dovepress.com/ testimonials.php to read real quotes from published authors. 